\title{
Plasma Processes in Pulsar Magnetospheres and Eclipsing Binary Pulsar Systems
}

\author{
Qinghuan Luo \\ Department of Physics \& Mathematical Physics \\ The University of Adelaide, SA 5005, Australia \\ Received on 30 March, 1998; Revised version on 17 August, 1998

\begin{abstract}
Plasma processes that may be responsible for pulsar radio emission and for eclipses observed for binary pulsars are discussed. High brightness temperature of pulsar radio emission implies that the radiation mechanism must be coherent. Several emission mechanisms are discussed. The high brightness temperature of radio emission also implies that nonlinear effect on wave propagation through pulsar magnetospheric plasmas is important and may result in radio pulse microstructure or cause fluctuation in dispersion measure. The discovery of eclipsing binary pulsars provides us with an opportunity to study nonlinear wave-wave interactions in electron-ion plasmas in the winds (or magnetospheres) of companion stars.
\end{abstract}

\section{Introduction}

Pulsars are thought to be rotating Tstrongly magnetized neutron stars Temitting radio waves. Radio emission from pulsars is in the form of periodic sequences of pulses like a light beam from lighthouse. The periods range from $10^{-3}$ to about 4 seconds [1]. The observed pulses also have secular variations which are usually described in terms of the period derivative (the time derivative of the period) $\Gamma$ typically in the range from $10^{-15}$ to $10^{-20}$. The observed pulse period can be associated with the rotation period of a neutron star and the secular change of the pulse period (increasing) can be associated with slowdown of the rotation. The slowdown can be attributed to the energy loss due to a large scale flux of electromagnetic energy or particle kinetic energy being carried away from the (magnetospheric) system (e.g. [2]). The current models for pulsars (e.g. [3FI5]) include (1) electron-positron pair production in polar region Tand (2) magnetospheres filled with electron-positron pair plasmas. A neutron star consists mainly of degenerated neutron gas and can be regarded as perfect conductor [6]. Rotation can induce an electric field which has a component parallel to the magnetic field and can accelerate particles to very high energies. These ultrarelativistic particles emit high energy photons through curvature radiation or synchrotron radiation (e.g. [7 [3]) or inverse
Compton scattering [819]. High energy photons initiate an electron-positron pair cascade $\Gamma$ which then limits the acceleration zone to a specific size [374519]. These outflowing pairs form a magnetosphere $\Gamma$ which are replenished continuously with electron-positron pairs produced through pair cascades by energetic particles accelerated in the acceleration zone.

A pulsar magnetosphere can be roughly divided into two zones: (1) open field line region near the magnetic pole Twhere pair plasmas are outflowing and pass through the light cylinder (where the co-rotation speed approaches the speed of light) Tand (2) closed field line regionTwhere pair plasmas are trapped. The region further outside the light cylinder is called the wind zone $\Gamma$ where plasmas move approximately radially. Observation of pulsar radio emission appears to suggest that emission is produced in the open field line regionTwhere electron-positron pair plasmas flow out along field lines (e.g. [14]). Investigation of plasma properties $\Gamma$ and in particular $\Gamma$ of radiation processes in that region is the main subject of interest in pulsar theory (e.g. [11П15]).

The main feature of magnetospheric pair plasmas is that the particle distribution in momentum space is highly anisotropic. Due to strong magnetic fields $\Gamma$ particles rapidly radiate away their perpendicular energy and move essentially along the magnetic field lines. Since electron and positron have the same mass $\Gamma$ 
when the plasma is neutral or quasineutral in charge $\Gamma$ some wave modes that appear in conventional electronion plasmas are modified and some even disappear (e.g. [11П2П15]; for discussion of nonrelativistic pair

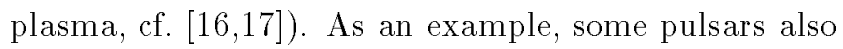
emit circularly polarized waves $\Gamma$ but for an electronpositron pair plasma with charge quasineutrality Wwave modes are mainly linearly polarized [11].

In the study of plasma processes relevant for pulsar radio emissionTthe following two areas have drawn considerable interest from researchers: production of coherent radio emissionTand propagation effects in the magnetospheric plasma. Despite a wide range of emission models have been proposed for coherent pulsar radio emission $\Gamma$ none of them can explain satisfactorily the observed properties of pulsar radio emission. HereГ I will concentrate on the basic plasma processes which include various types of plasma instabilities that may be relevant for pulsar radio emission and that have been explored in considerably details but are still not well understood in the context of pulsar radio emission.

Discovery of eclipsing binary pulsars provides us with opportunity to study nonlinear wave-wave interactions in electron-ion plasmas under the conditions that are more similar to laboratory plasmas. In such a system $\Gamma$ the pulsar and its companion are bound by gravitational force and orbit each other. Radio emission from the pulsar is periodically eclipsed by plasmas in the companion wind [18Г19Г20]. The plasma in the companion wind is possibly nonrelativistic and consists mainly of electrons and protons. When intense radio waves propagate through such a plasmaTnonlinear wave-wave interaction can be important and can even disperse the radio beamTresulting in eclipses.

In Sec. IIT coherent nature of pulsar radio emission is discussed. Dispersion properties of pair plasmas and relevant instabilities are discussed in Sec. III. The propagation effects on radio waves in pulsar magnetospheres are considered in Sec. IV. In Sec. V-VIIT eclipsing binary pulsars and the eclipse mechanism due to three-wave interaction are discussed.

\section{Pulsar radio emission}

The most important information that pulsar radio emission can immediately tell us is the coherent nature of radiation processes in pulsar magnetospheres. Although the observed flux density Ttypically within the range $10^{-3}$ to a few Jansky $\left(1 \mathrm{Jy}=10^{-26} \mathrm{Wm}^{-2} \mathrm{~Hz}^{-1}\right) \Gamma$ is relatively weak compared with other radio sources in astrophysics 5 due to the compactness of the source the inferred effective temperature is extremely high. Indeed $\Gamma$ since the typical duration of the pulse is about $10^{-3}$ s Tthe linear dimension of the source is smaller than $3 \times 10^{5} \mathrm{~m}$. The effective brightness temperature $T_{\text {eff }}$ can be defined by analogy with thermal emissionTfor which the specific intensity at low frequency is described by Rayleigh-Jeans law. For radio emission $\mathrm{T}$ the effective brightness temperature of a source region is defined by writing the radio intensity $\Gamma 2 \pi I_{\nu}\left(\mathrm{Wm}^{-2} \mathrm{~Hz}^{-1} \mathrm{sr}^{-1}\right) \Gamma$ in the form

$$
T_{\text {eff }}=\frac{2 \pi c^{2} I_{\nu}}{\nu^{2} \kappa}
$$

where $\kappa$ is the Boltzmann constant and $\nu$ is the radio frequency. The specific intensity $I_{\nu}$ can be related to the flux density $F_{\nu}$ by $I_{\nu}=F_{\nu} / \Delta \Omega_{0}$ Twhere $\Delta \Omega_{0}$ is the beam solid angle. As an example $\mathrm{T}$ for the Crab pulsar the mean flux density at $400 \mathrm{MHz}$ is about $F_{400} \approx$ $480 \mathrm{mJy}\left(=4.8 \times 10^{-27} \mathrm{Wm}^{-2} \mathrm{~Hz}^{-1}\right)$ and the distance is $D_{0} \approx 2 \mathrm{kpc}\left(=6.172 \times 10^{19} \mathrm{~m}\right)$ [1]. If the band width is taken to be $\Delta \omega \approx 400 \mathrm{MHz}$ Tone estimates that the radio luminosity is about $L_{r} \approx F_{\nu} D_{0}^{2} \Delta \nu \approx 10^{21} \mathrm{~J} \mathrm{~s}^{-1}$. If the linear size of the source region is $10^{4} \mathrm{~m}$ then the effective brightness temperature is estimated to be $T_{\text {eff }} \approx 3 \times 10^{27} \mathrm{~K}$.

For incoherent emission where particles radiate independently of each other $\Gamma$ thermodynamics implies that $\kappa T_{\text {eff }}$ be less than the kinetic energy of the radiating particles. For the brightness temperature as high as $T_{\text {eff }}=3 \times 10^{27} \mathrm{~K}$ Tto avoid self-absorptionT the radiating particles must have very high energy $10^{18} \mathrm{MeV}$ ! In

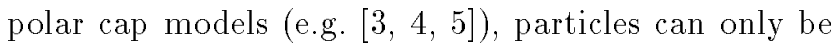
accelerated up to energies $\sim 10^{6}-10^{8} \mathrm{MeV}$. Therefore $\Gamma$ a coherent emission mechanism is required to produce radio emission with such an extremely high brightness temperature.

To explain the extremely high brightness temperature of pulsar radio emission $\mathrm{T}$ a large number of coherent emission mechanisms have been proposed. The majority of these models have remained at the stage of explaining the coherent nature i.e. to achieve the required high intensity $a$ and are not detailed enough to compare with observation. On the other handTthe current observational data are unable to tightly constrain modeling of pulsar radio emission. Emission models which have been explored in considerable details include coherent curvature emission by bunchingTcurvature maser emissionTlinear and nonlinear plasma insta- 
bilities (for a review $\Gamma \mathrm{cf}$. [21]). In the model of emission by bunches the size of the bunch of emitting particles is assumed to be smaller than the wavelengthTand then $\Gamma$ the phases of the spontaneous radiation fields by individual particles are coherent. The total radiation intensity then exceeds the sum of the spontaneous radiation intensities from each particle. For example $\Gamma N$ particles in a bunch can radiate up to $N^{2}$ times the radiation per individual particle. The main drawback of this type of model is that there is no satisfactory theory or model for producing and maintaining the particle bunches [29]. Thus This type of model will not be discussed further here $\Gamma$ and my following discussion will emphasize on those models based on maser emission or plasma instabilities.

\section{Electron-positron pair plasmas}

Dispersion properties of pair plasmas in pulsar magnetospheres can be derived using the one-dimensional approximation. In a pulsar magnetosphere $\mathrm{Cthe}$ particle motion can be separated into perpendicular and parallel parts with respect to the magnetic field lines. The perpendicular component of particle motion can be quantized into discrete energy levels called the Landau levels. The time-scale for a particle to lose all its perpendicular energy to synchrotron radiation and fall to the lowest Landau level is $t \sim 10^{-14}\left(10^{8} \mathrm{~T} / B\right)^{2}\left(\gamma / 10^{2}\right) \mathrm{s} \Gamma$ where $\gamma$ and $B$ are the Lorentz factor and the magnetic field $\Gamma$ respectively. For strong pulsar magnetic fields $\left(\sim 10^{8} \mathrm{~T}\right)$ Tthis time-scale is so short that all the particles should be in their lowest Landau levels and the particle motion in the pulsar magnetosphere (well within the light cylinder) is essentially one-dimensional.

The linear dispersion properties of a plasma can be described by permittivity tensor $K_{i j}=\delta_{i j}+$ $\left(i / \omega \epsilon_{0}\right) \sigma_{i j}$ with $\sigma_{i j}$ defined by writing induced current in terms of perturbed electric fields: $\delta J_{i}(\omega, \mathbf{k})=$ $\sigma_{i j}(\omega, \mathbf{k}) \delta E_{j}(\omega, \mathbf{k})$. The permittivity tensor for a strongly magnetizedTelectron-positron pair plasma can be derived in a way similar to that discussed by BaldwinTBernstein \& Weenink [22] except that there may be modification of vacuum polarization effect (e.g. [11Г23]) which is $\propto\left(\alpha_{f} / 4 \pi\right)\left(\hbar \Omega_{e} / m_{e} c^{2}\right)^{2}<1$ where $\alpha_{f}$ is the fine structure constant and $\Omega_{e}=10^{19} \mathrm{~s}^{-1}\left(B / 10^{8} \mathrm{~T}\right)$ is the nonrelativistic gyrofrequency. This quantum effect will be neglected in the following discussion of the permittivity tensor. In the one dimensional approximation $\Gamma$ Bessel function $J_{n}(z)$ in $K_{i j}$ can be expanded in $z=k_{\perp} c u_{\perp} / \Omega_{e} \Gamma$ where the particle momentum (in unit of $\left.m_{e} c\right) \Gamma$ wave vector are all separated into perpendicular and parallel components $\Gamma u_{\perp}$ and $u_{\|} \Gamma k_{\perp}$ and $k_{\|}$. To the lowest significant order in $z \Gamma$ and for $\left|\omega-k_{\|} c \beta_{\|}\right| \ll\left|\Omega_{e}\right|$ where $\beta_{\|}$is the parallel velocity (in c) Tone has

$$
\begin{gathered}
K_{11}=K_{22}=1-\sum_{s} \int d \gamma f_{s}(\gamma) \frac{\omega_{p s}^{2}}{\gamma \omega^{2}} \frac{\omega^{2}\left(1-n_{\|} \beta_{\|}\right)^{2}}{\omega^{2}\left(1-n_{\|} \beta_{\|}\right)^{2}-\Omega_{e}^{2} / \gamma^{2}}, \\
K_{12}=-K_{21}=\sum_{s} \int d \gamma f_{s}(\gamma) \frac{\omega_{p s}^{2}}{\gamma \omega^{2}} \frac{i \eta_{s} \omega\left(1-n_{\|} \beta_{\|}\right) \Omega_{e} / \gamma}{\omega^{2}\left(1-n_{\|} \beta_{\|}\right)^{2}-\Omega_{e}^{2} / \gamma^{2}}, \\
K_{13}=-K_{31}=\sum_{s} \int d \gamma f_{s}(\gamma) \frac{\omega_{p s}^{2}}{\gamma \omega^{2}} \frac{n_{\perp} \beta_{\perp} \omega^{2}\left(1-n_{\|} \beta_{\|}\right)}{\omega^{2}\left(1-n_{\|} \beta_{\|}\right)^{2}-\Omega_{e}^{2} / \gamma^{2}}, \\
K_{32}=-K_{23}=\sum_{s} \int d \gamma f_{s}(\gamma) \frac{\omega_{p s}^{2}}{\gamma \omega^{2}} \frac{-i \eta_{s} \omega n_{\perp} \beta_{\perp} \Omega_{e} / \gamma}{\omega^{2}\left(1-n_{\|} \beta_{\|}\right)^{2}-\Omega_{e}^{2} / \gamma^{2}}, \\
K_{33}=1-\sum_{s} \int d \gamma f_{s}(\gamma) \frac{\omega_{p s}^{2}}{\gamma \omega^{2}}\left[\frac{1}{\gamma^{2}\left(1-n_{\|} \beta_{\|}\right)^{2}}+\frac{\omega^{2} n_{\perp}^{2} \beta_{\perp}^{2}}{\omega^{2}\left(1-n_{\|} \beta_{\|}\right)^{2}-\Omega_{e}^{2} / \gamma^{2}}\right],
\end{gathered}
$$

where $\omega_{p s}$ is the plasma frequency $n_{\|}=k_{\|} c / \omega \Gamma n_{\perp}=$ $k_{\perp} c / \omega \Gamma \eta_{s}$ is the sign of the particle charge $\Gamma f_{s}(\gamma)$ is the particle distributionTand the sum is made over all particle species (electrons $\Gamma$ positronsTetc) and all components (including the beam components). Eq. (2ae) apply to uniform magnetic fields. When magnetic 
fields are inhomogeneous $\Gamma$ for example $\Gamma$ there is a spatial gradient or field line curvature $\Gamma$ particles have drift motion such as curvature drift $\Gamma$ which will be discussed in Sec. III.3.

The outflowing plasma consists of primary beams of electrons $\Gamma$ positrons or ions from polar caps and a background electron-positron pair plasma produced through pair cascades. Their densities can be estimated as follows. Suppose that pairs have average Lorentz factor $\gamma_{b} \Gamma$ primary particles have the Lorentz factor $\gamma_{p}$ Tand that the system can somehow adjust itself to equipartition $n_{ \pm} \gamma_{ \pm} \approx n_{b} \gamma_{b} \Gamma$ where $n_{ \pm} \Gamma n_{b}$ are the densities of pairs and primary particles $\Gamma$ respectively. Since the density of primary particles is the Goldreich-Julian density [10] Tgiven by $n_{b}=2 \epsilon_{0} \Omega B / e \approx$ $7 \times 10^{16} \mathrm{~m}^{-3}\left(B / 10^{8} \mathrm{~T}\right)(1 \mathrm{~s} / P)$ Tthe background plasma density is $n_{ \pm} \approx n_{b} \gamma_{b} / \gamma_{ \pm}$. The Lorentz factor of primary particles depends on the specific acceleration model $\Gamma$ and is less than $\gamma_{b, \max } \Gamma$ the value that can be achieved by acceleration through the potential drop across the polar cap. The specific form of distribution function for pair plasmas $\Gamma f_{ \pm}(\gamma) \Gamma$ depends on specific polar cap models. In Arons model [5] $\Gamma$ one has $f_{ \pm}(\gamma) \propto \gamma^{-1.5} \exp \left(-\gamma_{0} / \gamma\right)$. The distribution has a peak at $\gamma_{0}=50-100$ (the value depends on other physical parameters as wellГcf. [5]).

Using (2a-e) Tone can solve Maxwell's equations to derive dispersion relations for various types of waves. In the strong field approximation $B \rightarrow \infty \Gamma(2 \mathrm{a}-\mathrm{e})$ are simplified to $K_{i j} \approx 0$ for $i \neq j \Gamma K_{i j} \approx 1$ for $i=j=1 \Gamma$ $2 \Gamma$ and $K_{33} \approx 1-\Delta K$ where

$$
\Delta K=\sum_{s} \frac{\omega_{p s}^{2}}{\omega^{2}} \int \frac{d \gamma f_{s}(\gamma)}{\gamma^{3}\left(1-n_{\|} \beta_{\|}\right)^{2}} .
$$

Then The dispersion relation reduces to $n=1$ for extraordinary mode $\Gamma$ and

$$
\left(1-n_{\|}^{2}\right)(1-\Delta K)-n_{\perp}^{2}=0,
$$

for ordinary mode $\Gamma$ where propagation of the ordinary mode waves strongly depends on the angle between $\mathbf{k}$ and $\mathbf{B}$ (magnetic field). When $\mathbf{k} \| \mathbf{B}$ Tthe waves split into two types. The first type is transverse $\Gamma$ with refractive index equal to unity. The second type is a Langmuir wave. According to their phase speed $\Gamma$ the ordinary mode waves described by (4) have two branches: superluminal waves whose phase speed is faster than $c$ Tand subluminal waves $\Gamma$ whose phase speed is slower than $c$. In the approximation described above $\Gamma$ instabilities as the result of the lowest order wave-particle interaction can occur only for subluminal branch of ordinary mode since these waves have phase speed less than $c$ and the Cerenkov resonance condition can be satisfied.

In the region near the polar cap $\Gamma$ the plasma density is so dense that the condition $\omega / \omega_{p} \ll 1$ is satisfied. Then Tone has a solution of subluminal waves with the dispersion relation given by

$$
\omega^{2}=k_{\|}^{2} c^{2}\left(1-\frac{k_{\perp}^{2} c^{2}}{8\langle\gamma\rangle \omega_{p}^{2}}\right)
$$

where $\langle\gamma\rangle$ is the average Lorentz factor of the pair plasma. Waves described by (5) are also called (modified) Alfvén waves (e.g. [15]).

Various linear plasma instabilities were considered for coherent pulsar emissionTand these can be broadly classified into three types $\Gamma$ which include instabilities for (a) electrostatic waves $\Gamma$ e.g. Langmuir waves $\Gamma$ (b) electrostatic-electromagnetic waves $\Gamma$ e.g. modified Alfvén waves given by Eq. $5 \Gamma$ which have both electrostatic and electromagnetic components $\Gamma$ and (c) transverse waves $\Gamma$ e.g. cyclotron instability $\Gamma$ which is electromagnetic and can escape directly to interstellar medium.

\section{III.1 Two-stream Instability}

A widely-discussed instability for electrostatic waves in the context of pulsar emission is the two-stream stability [3133125126127129]. Pulsar magnetospheric plasmas can be regarded as a beam-plasma system since they consist of a pair plasma and energetic electron or positron (or ion) beams. In plasma theory it is wellknown that such a system is unstable to the development of what is often called two-stream instability [24]. There are two types of two-stream instabilities $\Gamma$ the counter-streaming instability and the weak beam instability. The counter-streaming instability is due to two components of the plasma counter streaming through each other $\Gamma$ with the same density and opposite velocities. The weak beam instability is due to a beam traveling through a background plasmaTwhere the beam is less dense than the background so that the wave modes are determined by the background and interact with the 
fast beam. The underlining physics of these two types of instabilities is similarTthat isTinstabilities occur due to that beam particles satisfy the Cerenkov resonance condition.

The two-stream instability normally occurs for electrostatic waves. Therefore $T$ the instability itself can not produce radiation directly. Ruderman \& Sutherland [3] proposed that the instability of such a beam-plasma system can result in particle (electron and positron) bunching $\Gamma$ and that the bunched particles can radiate in phase to produce coherent curvature emission. HoweverTBenford \& Buschauer [25] concluded that the growth rate of such an electrostatic instability is not sufficient to explain the level of the pulsar radio emission (also [26 [27[29]).

Cheng \& Ruderman [33] suggested that the counter-streaming instability with a relatively larger growth rate (compared with Ruderman \& Sutherland's model [3]) may occur due to relative motion of the electrons and positrons of the pair plasma. The relative motion of the electrons and positrons is due to the presence of the primary beam and the rotation of the magnetosphere. HoweverTthe counter-streaming instability may not be effective if the electrons and positrons of the pair plasma have a broad distribution of parallel momenta.

\section{III.2 Cyclotron Instability}

When terms $\propto 1 /\left(\omega-k_{\|} v_{\|} \pm \Omega_{e} / \gamma\right)$ are retained in $(2 \mathrm{a}-\mathrm{e}) \Gamma \mathrm{cyclotron}$ instability may occur. Consider energetic particles in the primary beam or in the tail of the distribution of the secondary pair plasma. It has been suggested that these particles may satisfy the anomalous doppler resonance condition $\Gamma$ allowing a cyclotron instability to develop [34]. The anomalous doppler effect and the associated instability can be understood as follows. On emission of a photon $p_{\|}$changes to $p_{\|}-\hbar k_{\|}$through conservation of momentum and the particle energy $\varepsilon=\left(m^{2} c^{4}+p_{\|}^{2} c^{2}+2 n_{L} e B \hbar c\right)^{1 / 2}$ (where the spin effect is ignored and $n_{L}$ is the Landau levels) changes to $\varepsilon-\hbar \omega$ by conservation of energy. Writing $\varepsilon-\hbar \omega=\left[m^{2} c^{4}+\left(p_{\|}-\hbar k_{\|}\right)^{2} c^{2}+2 n_{L}{ }^{\prime} e B \hbar c\right]^{1 / 2}$ and $l=n_{L}-n_{L}{ }^{\prime} \Gamma$ and then taking $\hbar \rightarrow 0$ one derives the doppler condition $\omega-k_{\|} v_{\|}-l \Omega_{e} / \gamma=0$. The normal doppler effect corresponds to $l>0 \Gamma$ and the anomalous doppler effect corresponds to $l<0$ [23Г35]. The physics of the cyclotron instabilityCi.e. the anomalous doppler effect $\Gamma$ is that the parallel energy serves as free energy such that a particle can radiate a photon while transiting from a lower level $n_{L}$ to an excited level $n_{L}{ }^{\prime} \Gamma$ viz. $l<0$. The parallel energy decreases more than the perpendicular energy increases $\Gamma$ allowing overall energy conservation. In the case of pulsars 5 since $B$ is large Tone needs only to consider the transition between $n_{L}=0$ and $n_{L}^{\prime}=1$. Then the anomalous doppler condition can be rewritten as $\omega-k_{\|} v_{\|}+\Omega_{e} / \gamma=0$.

Because of the very strong magnetic field in pulsar magnetospheres $\Gamma$ the cyclotron instability can develop only near or beyond the light cylinder. HoweverTobservation favors the suggestion that emission comes from the region well inside the light cylinder [14].

\section{III.3 Instability induced by curvature drift}

Instabilities may occur for electrostaticelectromagnetic waves described by (5) when an energetic beam traveling through the dense background electron-positron pair plasma in a magnetic field with curved field lines. When magnetic field lines have curvature Telectrons or positrons can drift across field lines with the drift speed $v_{d}=v_{\|}^{2} \gamma /\left(\Omega_{e} R_{c}\right)$ where $v_{\|}$is the parallel (to $\mathbf{B}$ ) velocity $R_{c}$ is the radius of field line curvature [36Г $37 \Gamma 38]$. The cyclotron terms in (2a-e) are modified to $\omega-k_{\|} v_{\|}-k_{\perp} v_{d} \pm \Omega_{e} / \gamma$. One expects that the 'hydrodynamic' instability due to curvature drift can occurT since the inclusion of curvature drift modifies the Cerenkov resonance condition. In the case of uniformly magnetized plasmaT the Cerenkov resonance condition corresponds to parallel phase velocity of waves equaling the parallel velocity of the particles (when the particles move along the field lines). Therefore the resonance condition is symmetric about the field line directionTi.e. it corresponds to the surface of the Cerenkov cone (about the magnetic field line) defined by the wave vector. In the presence of curvature drift the resonance condition requires that the parallel phase velocity of the waves be either larger or smaller than the parallel velocity of the particles $\Gamma$ and this implies that the resonance condition depends explicitly on the signs of both the particle charge and the viewing angle (the angle between the field line and the wave vector). This feature can result in growth 
of the waves with dispersion relation $(5) \Gamma$ which have significant transverse component.

Other form of coherent curvature emission involving field line curvature is called curvature maser emissionI which relies on an effective particle population inversionTi.e. number of particles with higher energy is significantly larger than those with lower energies [37 I39]. This type of distribution can be the source of free energy to sustain the maser emission (negative absorption). The growth of waves can be described by absorption coefficient which can be calculated using Einstein coefficient method [23].

\section{III.4 Nonlinear interaction}

Apart from linear plasma instabilitiesTplasma processes involving nonlinear interaction can be important in pulsar radio emission. There are two main reasons why nonlinear instabilities should be considered as well: (a) an instability initially in the linear regime $\Gamma$ may grow to the nonlinear regime $\Gamma$ and (b) electromagnetic radiation can be produced through conversion of other types of wave $\Gamma$ e.g. electrostatic waves. For (a) $\Gamma$ when the amplitude of waves as the result of instability exceeds a critical value $\Gamma$ the variation of the zero-order orbit of the particles becomes important and the energy transfer between the fast particles and the waves can be oscillatory. Therefore Tnonlinear effects must be considered.

There are possibilities that strong Toscillating electric fields are generated near polar caps $\Gamma$ e.g. as the result of the polar gap oscillation [28] Tthough no detailed models have been developed yet. AlternativelyГelectrostatic waves can be produced through beam streamingTbut its effectiveness of growth remains unclear (e.g. Sec. III.1). If strong electrostatic waves exist near polar capsTelectromagnetic waves can be produced through either nonlinear interaction or induced scattering. A model based on coherent emission by particles accelerated in a large amplitude $\Gamma$ oscillating electric field was proposed by Melrose [29] Tand further discussed by Rowe [30Г31]. In the modelГelectromagnetic radiation is produced through induced scattering of electrostatic waves (which can be assumed to be superluminal) by relativistic particles.

Asseo et al. [32] proposed a model based on electromagnetic radiation by Langmuir solitons. In their
modelCLangmuir turbulence is assumed to be present $\Gamma$ e.g. due to two-stream instability (cf. Sec. III.1) Tand the perpendicular component of electric field of the soliton can be excited as the result of irregularities in the perpendicular direction. The parallel electric field acts as a source of radiation. An advantage of the model is that the mechanism can produce pulse microstructure (cf. Sec. IV). However $\Gamma$ an effective mechanism for generating Langmuir turbulence is required for the model.

\section{Propagation of pulsar radio emission}

If radio emission is produced well inside the light cylinder as apparently favored by observation (e.g. [14]) $\Gamma$ the intense radio waves must propagate through the magnetospheric plasmaTand the propagation effect on radio waves within the magnetosphere can be significant. One of the important aspects of propagation effects is refraction of raysTi.e. propagation direction changes because of inhomogeneity or anisotropy of plasmas.

\section{IV.1 Refraction of rays in an anisotropic plasma}

The standard description of refraction rays is the geometric optical approximation $\Gamma$ in which the characteristic length scale is much larger than the wavelength $c / \omega$. In this approximation the rays (propagation of waves) are described by the Hamilton form: $d \mathbf{x} / d t=\partial \omega / \partial \mathbf{k} \Gamma d \mathbf{k} / d t=-\partial \omega / \partial \mathbf{x} \Gamma$ where $\mathbf{k}$ is the wave vector $\Gamma t$ is a parameterized distance along the ray path $\Gamma$ and $\omega(\mathbf{k}, \mathbf{x})$ is the dispersion in a locally homogeneous plasma.

An example of bending of rays in the pulsar magnetospheric plasma was discussed by Melrose [11]. He specifically considered the low-density limit Tin which there are two natural modes with the refraction indices close to 1 . Because of magnetic fields $\Gamma$ the two modes propagate in a different mannerTand initially two identical rays of the two natural modes will split. Let $n_{1} \Gamma n_{2}$ be the refraction indices of extraordinary and ordinary modes. In the strong magnetic field limitTassuming the plasma is neutral and symmetric $\Gamma$ we have $n_{1}=1$. The 
index of ordinary mode can be derived from (3). One then has

$$
n_{2}-n_{1} \approx \frac{\omega_{p}^{2} \sin ^{2} \theta}{\gamma^{2} \omega^{2}(1-\beta \cos \theta)^{2}},
$$

where $\omega \gg \omega_{p} / \gamma$ and we assume the cold plasma approximation. The angular separation of the two rays is $\Delta \Theta=\partial\left(n_{2}-n_{1}\right) / \partial \theta \Gamma$ that is $\Gamma$

$$
\Delta \Theta=\frac{8 \omega_{p}^{2} \theta}{\omega^{2}} \frac{1+3 \gamma^{2} \theta^{2}}{\left(1+\gamma^{2} \theta^{2}\right)^{2}} .
$$

In the low-density approximation Twe have $\Delta \Theta \ll 1$ for $\theta \ll 1$. The angular separation strongly depends on the frequency.

\section{IV.2 Nonlinear dispersion}

Radio waves of different frequencies travel through plasmas with different group velocitiesTand the difference between the propagation time is an integration of $\left(1 / \beta_{g 1}-1 / \beta_{g 2}\right)$ along $D \Gamma$ where $\beta_{g 1}$ and $\beta_{g 2}$ are the group speeds in $c \Gamma$ and the two radio frequencies are $\omega_{1}$ and $\omega_{2}$. Let $D_{*}$ and $D_{0}$ be the distances of the source region and the observer to the star's center $\Gamma$ respectively. One may define the dispersion measure

$$
D M \equiv \int_{D_{*}}^{D_{0}} \frac{n_{p} d D}{\left(\omega_{p} / \omega_{1}\right)^{2}-\left(\omega_{p} / \omega_{2}\right)^{2}}\left(\frac{1}{\beta_{g 1}}-\frac{1}{\beta_{g 2}}\right) .
$$

For an unmagnetized Tnonrelativistic plasma with linear dispersion relation $n^{2}=\left(1-2 \omega_{p}^{2} / \omega^{2}\right)$ Tone has the group velocity $v_{g}=c\left(1-\omega_{p}^{2} / \omega^{2}\right)$. In this case $\Gamma$ the dispersion measure is $(D M)_{l}=\int_{D_{*}}^{D_{0}} n_{e} d D \Gamma$ where the subscript $l$ denotes the usual linear dispersion measure. For ISMTcyclotron frequency is much less than the radio frequency and (8) is then applicable. In practice $\Gamma$ dispersion measure (8) is often used to estimate pulsar distance provided that electron density of ISM is given or vice versa [1].

Due to high brightness temperature of pulsar radio emission $\Gamma$ nonlinear effects on wave propagation may modify dispersion measure [43]. A nonlinear effect can be characterized by a dimensionless $\Gamma$ Lorentz invariant parameter $\beta_{Q}=e E /\left(m_{e} c \omega\right) \Gamma$ where $E$ is the electric field of radio emission $\Gamma \omega$ is the radio frequency. Assuming that the source size is $R_{\star} \Gamma$ and the luminosity $\left(\mathrm{J} \mathrm{s}^{-1}\right)$ of radio emission is $L_{*} \sim \epsilon_{0} E^{2} c R_{*}^{2}$. ThenTone has $\beta_{Q} \approx\left(c / R_{*} \omega\right)\left[\left(4 \pi L_{*} / m_{e} c^{2}\right)\left(r_{e} / c\right)\right]^{1 / 2} \approx$ $14.3\left(L_{*} / 10^{21} \mathrm{~J} \mathrm{~s}^{-1}\right)^{1 / 2}(400 \mathrm{MHz} / \nu)\left(10^{4} \mathrm{~m} / R_{*}\right)$ where $r_{e}=e^{2} / 4 \pi \epsilon_{0} m_{e} c^{2} \approx 2.8 \times 10^{-15} \mathrm{~m}$ is the classical electron radius $\Gamma$ the refraction index is $n \approx 1 \Gamma$ and $\nu=\omega / 2 \pi$. As waves propagate away from the source region $\Gamma \beta_{Q}$ decreases as $\beta_{Q} \propto 1 / D$ where $D$ is the distance of the relevant region to the star's center $\left(R_{*}\right.$ in $\beta_{Q}$ is replaced by $D$ ).

Dispersion measure including nonlinear effect can be derived by evaluation of $\beta_{g 1}$ and $\beta_{g 2}$ using the nonlinear dispersion relation $n^{2}=1-2 \omega_{p}^{2} /\left(\gamma_{Q} \gamma_{ \pm} \omega^{2}\right) \Gamma$ where $\gamma_{ \pm}$is the Lorentz factor of pair plasma and where $\gamma_{Q}=\left(1+\beta_{Q}^{2}\right)^{1 / 2}$ (e.g. [40Г41Г42]). Since the nonlinear dispersion relation depends on the intensity of radio emissionTany temporal fluctuation in the intensity can result in fluctuation in dispersion measure $\Gamma$ which may be potentially observable.

For illustrative purposes $\Gamma$ here we consider the approximation $\nu>\Omega_{e} / 2 \pi \Gamma$ which may not be a good approximation in the region deep inside the magnetosphere. Using Eq. (8) Г one finds the fluctuation in dispersion measure due to nonlinear dispersion $\Gamma$ that is $\Gamma$

$$
(D M)_{n} \approx\left(\frac{\gamma_{b}}{\gamma_{ \pm}^{2}}\right) n_{b} R_{0} I=2.25 \times 10^{-2}\left(\frac{1 \mathrm{~s}}{P}\right)\left(\frac{B_{0}}{10^{12} \mathrm{G}}\right)\left(\frac{\gamma_{b}}{\gamma_{ \pm}^{2}}\right) I\left(\mathrm{~cm}^{-3} \mathrm{pc}\right)
$$

where $\gamma_{b}$ is the Lorentz factor of the primary electrons (or positrons) $\Gamma I \approx 1 /\left(\beta_{Q} x_{s}^{2}\right) \approx 4.4 \times 10^{-8} / \beta_{Q}$ with $x_{*} \approx R_{L} / R_{0} \approx c /\left(R_{0} \Omega\right) \approx 4.78 \times 10^{3} \Gamma R_{L}=c / \Omega$ is the radius of the light cylinder $\Gamma R_{0}$ is the star's radius $\Gamma n_{b}$ is the G-J density as defined earlier. (Compared to the re- sult derived by Wu \& Chian [43] Tthe right-hand side is smaller by factor of $\gamma_{ \pm}^{2}$.) Assuming $\gamma_{b}=10^{7} \Gamma \gamma_{ \pm}=10 \Gamma$ we obtain $(D M)_{n} \approx 10^{-4}\left(\mathrm{~cm}^{-3} \mathrm{pc}\right) \Gamma$ which is superposed to the usual linear dispersion measure (due to ISM). The observational consequence of the fluctuation 
due to nonlinear dispersion was discussed in details by Wu \& Chian [43].

\section{IV.3 Modulational instabilities}

Observation of individual pulses shows intensity variations over very short time scaleTtypically microseconds [44]. This phenomena is called the pulse microstructure. Chian \& Kennel first suggested that intense radio waves propagating through magnetospheric plasmas may undergo modulational instability may provide a mechanism for causing pulse microstructure [45]. In nonlinear plasma theory that amplitude modulation of waves of frequency $\omega / 2 \pi$ over time scale much less than $2 \pi / \omega$ can be unstable if the group dispersion $P=0.5 \partial v_{g} / \partial k=0.5 \partial^{2} \omega / \partial k^{2}$ and the nonlinear frequency shift $Q=-\partial \omega / \partial|A|^{2}$ satisfy the condition $P Q>0 \Gamma$ where $v_{g}$ is the group velocity $A(\mathbf{x}, t)$ is the envelope of modulated wave amplitude $\Gamma$ the wave vector potential is expressed as $A(\mathbf{x}, t) \exp [i(\mathbf{k} \cdot \mathbf{x}-\omega t)]+c . c($ e.g. [46]). In general $\Gamma$ the envelope $A$ is determined by the nonlinear Schrödinger equation. Thus $\Gamma$ the slow modulation $A$ can be regarded as quasiparticles in the sense that they are described by the wave function $\Gamma \Gamma$ and the instability can be interpreted as bunching of these quasiparticles towards the potential well. When the condition is satisfied $\Gamma$ the quasiparticles bunching enhances the potential well and attracts more quasiparticlesT resulting in a self-modulation instability [46].

Initial growth of modulational instability can be treated as perturbation; a dispersion relation for modulation can be derived in close analogy with linear analysis of wave instabilities except that the dispersion relation depends on the large wave amplitude [45Г47Г48 51Г52]. The growth of modulational instability requires that the amplitude exceeds the threshold (i.e. the amplitude must be large enough to allow the instability to occur). From the dispersion relation the growth rate of modulational instability can be calculated.

Nonlinear analysis of wave propagation in electronpositron plasmas was discussed by Chian \& Kennel [45] I Kates \& Kaup [49] Tand recently by Gratton et al. [50].
There was similarTearlier work by Sakai \& Kawata [13] on nonlinear propagation of Alvén waves in ultrarelativistic electron-positron plasmas. In general $\Gamma$ the full solution of nonlinear Shrödinger equation leads to solitons or turbulence $\Gamma$ and the nonlinear solution reduces to the result of linear analysis in the weak modulation limit. The problem of nonlinear propagation of electromagnetic waves was also studied by several other authors $\Gamma$ e.g. the case with magnetic fields was considered by StenflorShukla \& Yu [55] TMofiz et al. [54] Tthe case with ion components was discussed by Rizzato [56] $\Gamma$ Rizzato $\Gamma$ Schneider \& Dillenburg [57] $\Gamma$ and the case of relativistic plasmas was studied by MikhailovskiiTOnishchenko \& Tatarinov [58] TMofiz [53].

\section{Eclipsing binary pulsars}

Several eclipsing binary pulsars have been discovered. Among these eclipsing binary pulsar systems $\Gamma$ both PSR B1957+20 and PSR B1744-24A are millisecond pulsars with short orbital period and a lowmass companion [59Г19]. (Eclipsing binary pulsar PSR J2051-082 was recently discovered to have similar parameters to PSR B1957+20 [79180]) The observed properties of these two eclipsing pulsars can be summarized as follows. (1) The eclipse radius is larger than the inferred Roche lobe radius and is much larger than the radius of the companion. (2) The eclipse radius is frequency dependent $\Gamma R_{E} \sim \nu^{-\delta}$ with $\delta \sim 0.41$ for PSR B1957+20 and $\delta \sim 0.63$ for PSR B1744-24A. (3) The propagation time of the pulsar signal at egress (exit of eclipse) is longer than at ingress (entrance of eclipse). (4) Eclipsing is approximately symmetric about orbital phase 0.25 at which the pulsar is behind the companion. (5) There are continuum eclipses at lower frequencies. For PSR B1957+20Гthe average continuum flux density at $318 \mathrm{MHz}$ was observed to drop dramatically during pulsed eclipse $\Gamma$ while the $1.4 \mathrm{GHz}$ continuum flux density was almost unchanged [60]. For PSR B1744-24AT the similar continuum or partial eclipses were observed for frequencies below $1.6 \mathrm{GHz}$. All these properties indicate that the eclipsing is due to the plasma of the companion winds rather than the material inside the 
Roche lobe.

\section{V.1 Plasma conditions in eclipse region}

Through accurate timing Tone of the most remarkable advantages of pulsar observationsTand optical observation of the companionTwe can estimate in details some important physical parameters that characterize the plasma conditions near or in the eclipse region. The plasma density can be estimated from the observed delays in propagation time of pulsar signal. The propagation time increases with increasing plasma density and decreases with increasing frequency. Near the eclipse region the plasma density increases with decreasing radial distance from the companion starTand this causes excessive time delays for radio waves traveling through the plasma. For PSR B1957+20 Tthe observed delays in propagation time give the electron column density near the ingress of about $4 \times 10^{19} \mathrm{~m}^{-2}$ at $318 \mathrm{MHz}$ [61]. If the characteristic length of the eclipsing material along the line of sight is $\sim R_{E}=0.68 R_{\odot}=4.7 \times 10^{8} \mathrm{~m}$ at $318 \mathrm{MHz}[59] \Gamma$ the plasma density is estimated to be $10^{11} \mathrm{~m}^{-3} \Gamma$ corresponding a plasma frequency of $\nu_{p}=\omega_{p} / 2 \pi=5.2 \mathrm{MHz}$.

The average parallel (to the line of sight) magnetic field can be estimated by measuring the delay between the right and left circularly polarized signals. For PSR B1957+20 The inferred $B$ is $\sim 10^{-4} \mathrm{~T}$ [59]. The presence of much stronger field is possible further inside the eclipse region (e.g. [62]).

The plasma temperature of eclipsing plasmas can be as high as $\sim 10^{6} \mathrm{~K}$ [59Г60]. For a companion star with a mass of a few per cent of a solar mass Tas in PSR B1957+20 and PSR B1744-24A Athe minimum temperature is $>10^{6}$ KTwhich may be estimated by equating the gravitational potential at the surface to the electron thermal energy. The optical observations also suggest that the outflowing plasma from the companion is sustained and heated by the pulsar wind [63Г64].

\section{V.2 Eclipse mechanisms}

Although there are extensive observational data on eclipsing pulsars $\Gamma$ PSR B1957+20 [59 61$]$ and PSR

B1744-24A [66 67$] \Gamma$ two fundamental issues remain unsolved: what physical process causes the eclipse Tand how the pulsar wind interacts with the companion star. Several mechanisms have been proposed to explain the eclipses. These include (1) refractive/reflection model (e.g. [68])Г (2) absorption models $\Gamma$ e.g. free-free absorption [69.70171] and cyclotron absorption [72] Гand (3) induced scattering models $\Gamma$ e.g. Raman scattering [73174172162] and Brillouin scattering [78] Tin which the eclipse is attributed to nonlinear wave-wave interactions in the plasma that lead to an effective scattering of the beam of pulsar radio emission. For both the systems the inferred plasma frequency is far below the observed frequency and this in fact rules out refraction/reflection as the cause for eclipses. Moreover $\Gamma$ the refraction/reflection model cannot predict a correct frequency dependence of eclipse duration. Among the absorption models Tthe free-free absorption requires rather a cool wind with temperature $\sim 300 \mathrm{KT}$ which appears implausible in view of the strong irradiation from the pulsar wind. The cyclotron absorption model by Thompson et al. [72] requires a strong magnetic field and a very hot wind with $T_{e}>10^{8} \mathrm{~K}$. This type of model predicts much stronger frequency dependence than that inferred from the observations.

Among these proposed mechanisms $\Gamma$ induced scattering appear the most plausible for pulsar eclipses. The arguments in favor of this include (1) pulsar radio emission has a high brightness temperature Tand (2) the radio emission is highly beamed. Condition (1) favors nonlinear interactions of radio waves with other lowfrequency waves in the plasma. Condition (2) implies that the radio emission can be regarded as a photon beam $\Gamma$ and such a beam can produce an instability in which the low-frequency waves grow due to the nonlinear interaction. The low-frequency waves can then scatter the high-frequency photon beam $\Gamma$ resulting in diffusion of the photon beam in $\mathbf{k}$-space $\Gamma$ and thus producing the eclipse.

\section{Three-wave interactions}

In the random phase approximation Tthe radio beam can be modeled as a collection of photonsTwhose distribution in the wave vector space is confined to a small solid angle and described by the occupation number 
$N(\mathbf{k})$ Twhose integration over $\mathbf{k}$ gives the photon density. The nonlinear interaction is described by a set of kinetic equations of $N(\mathbf{k})$ [75Г 76]. The evolution of $N(\mathbf{k})$ is assumed to be much slower than the reciprocal of the lowest frequency of all relevant wavesTand thus $\Gamma$ the weak turbulence theory is applicable [76].

The waves in a three-wave interaction satisfy the beat conditions

$$
\omega(\mathbf{k})=\omega^{\prime}\left(\mathbf{k}^{\prime}\right) \pm \omega^{\prime \prime}\left(\mathbf{k}^{\prime \prime}\right), \quad \mathbf{k}=\mathbf{k}^{\prime} \pm \mathbf{k}^{\prime \prime}
$$

which correspond to energy and momentum conservations in the semiclassical formalism.

\section{VI.1 Small angle scattering}

In the small angle scattering approximation $|\mathbf{k}| \sim$ $\left|\mathbf{k}^{\prime}\right| \gg\left|\mathbf{k}^{\prime \prime}\right| \Gamma \omega \sim \omega^{\prime} \gg \omega^{\prime \prime} \Gamma$ the kinetic equations reduced to a pair of equations [35177162178]

$$
\begin{gathered}
\frac{d N^{L}}{d t}=-\Gamma N^{L}+S^{L}, \\
\frac{d N}{d t}=\frac{\partial}{\partial k_{i}}\left(D_{i j} \frac{\partial N}{\partial k_{j}}+G_{i} N^{2}\right),
\end{gathered}
$$

where the sum over the repeated subscript indices $i \Gamma$ $j$ is implied and where $N$ and $N^{L}$ represent the photon and low-frequency wave occupation numbers $\Gamma$ respectively. The first equation describes two effects on the low-frequency waves: absorption (or instabilities) with the absorption coefficient $Г Г$ and the production of low-frequency waves through induced photon decay described by $S^{L}$. Note that $S^{L}$ is the counterpart of "spontaneous emission" in wave-particle interaction but here it is $\Gamma$ in fact $\Gamma$ the induced processes $\Gamma$ e.g. [35]. When $\Gamma<0$ Tinstabilities occur. All these quantities are related to three wave probability $w\left(\mathbf{k}, \mathbf{k}^{\prime \prime}\right) \Gamma$ which describes the probability of emission of low-frequency wave with $\mathbf{k}^{\prime \prime}$ by a high-frequency photon with $\mathbf{k}$. The calculation of $\Gamma$ is analogous to that for the absorption coefficient for waves due to resonant interaction with particles. The high-frequency photons play the role of the particles with the particle distribution function $f(\mathbf{p})$ Treplaced by the photon occupation number $N$. Just as particle-wave interaction can lead to instability of the low-frequency waves under the appropriate conditions $\Gamma$ so the three-wave interactions can lead to a photon-beam-induced instability $\Gamma$ described here by $\Gamma<0$.

Scattering effects on the high-frequency photon beam by low-frequency waves is described by diffusion coefficient $D_{i j}$. The diffusion of the photon beam is similar to diffusion of a particle beam due to waveparticle interaction [77Г62]. The quantities $\Gamma \Gamma D_{i j} \Gamma S^{L}$ and $G_{i}$ can be derived using the method discussed by Melrose [35Г 74] ГLuo \& Melrose [77Г62].

\section{VI.2 Large angle scattering}

For large angle scattering Tone needs to consider the full kinetic equations $\mathrm{W}$ which take the following form

$$
\begin{gathered}
\frac{d N_{ \pm}^{L}}{d t}=\dot{N}_{\mathrm{IPD} \pm}^{L} \mp \Gamma_{ \pm} N_{ \pm}^{L}, \\
\frac{d N}{d t}=\int d \Omega^{\prime} W\left[N\left(\mathbf{k}^{\prime}\right)-N(\mathbf{k})\right]\left(N_{+}^{L}+N_{-}^{L}\right),
\end{gathered}
$$

where $W=\omega^{2} \bar{u} / 4 \pi^{2} c^{3}$ and

$$
\bar{u}=(2 \pi)^{3} \hbar R^{\prime \prime}\left|e_{i}^{*} e_{j}^{\prime} e_{l}^{\prime \prime} \alpha_{i j l}\right|^{2} /\left(\omega \omega^{\prime} \omega^{\prime \prime}\right) .
$$

The ratio of the electric to the total energy in lowfrequency waves is represented by $R^{\prime \prime}$. The quadratic response tensor is given by $\alpha_{i j l} \Gamma$ and polarization is given by $e_{i}$ for high-frequency waves $\Gamma e_{j}^{\prime}$ for scattered high-frequency waves with frequency $\omega^{\prime} \Gamma e_{l}^{\prime \prime}$ for lowfrequency waves with frequency $\omega^{\prime \prime}$. Calculation of $\bar{u}$ is given in Luo \& Melrose [62]. In (14) $\Gamma N_{ \pm}^{L}$ represents the occupation number of low-frequency waves $\Gamma$ where the plus sign corresponds to the low-frequency waves emitted by scattering high-frequency photons from $\mathbf{k}$ to $\mathbf{k}^{\prime}$ $\left(\omega>\omega^{\prime}\right) \Gamma$ and the minus sign corresponds to the lowfrequency waves being absorbed $\left(\omega<\omega^{\prime}\right)$. Integration is made over the solid angle of $\mathbf{k}^{\prime}$. The term $\dot{N}_{\text {IPD }}^{L}$ corresponds to production of low-frequency waves through induced photon decay $\Gamma$

$$
\dot{N}_{\mathrm{IPD} \pm}^{L}=\int d \Omega W D_{1} D_{2} N\left(\mathbf{k} \mp \mathbf{k}^{\prime \prime}\right) N(\mathbf{k}),
$$

with $D_{1}=\left|\mathbf{e} \cdot \mathbf{e}^{\prime}\right|^{2} \Gamma D_{2}=1-\mathbf{k} \cdot \mathbf{k}^{\prime} / k k^{\prime}$.

\section{VI.3 Application to pulsar eclipses}

Small angle scattering is applicable for unmagnetized plasmas. The relevant low-frequency waves are 
plasma waves. For the parameters appropriate for eclipsing binary pulsars $\Gamma$ Landau damping constrains on $\mathbf{k}^{\prime \prime}$ and one has $\left|\mathbf{k}^{\prime \prime}\right| \ll|\mathbf{k}| \sim\left|\mathbf{k}^{\prime}\right|$. For small angle scattering the estimated growth rate for low-frequency waves is quite large [62]. The energy density in the low-frequency waves can grow to the saturated level provided that the damping rate is slow. The saturated level can be controlled by the perpendicular diffusion whose effects are to reduce the angular anisotropy of the photon beam. The characteristic time for perpendicular diffusion is $t_{\perp} \sim D_{i i} / k_{\perp}^{2}$ with $k_{\perp} \approx k \theta_{0}$. We assume that the photon beam has an angular width $\theta_{0}$. For the maximum growth $\Gamma_{\max } \Gamma$ one has $t_{\perp} \sim 1 / \Gamma_{\max }$. In the application to PSR B1957 + 20 and PSR B1744-24AT small angle scattering model appears to predict more strong frequency dependence of eclipse radius than that inferred from observation.

For magnetized plasmas which may be the case in eclipsing binary pulsars Tlarge angle scattering involving Bernstein waves may occur. For large angle scattering $\Gamma$ one may estimate the scattering effect by calculating $\Gamma=-(1 / N) d N / d t$. The scattering effect is important if the optical depth $\tau \approx \Gamma R_{E} / c$ is larger than 1. Possible application of large angle scattering involving lowfrequency Bernstein waves was considered by Luo \& Melrose [62].

\section{Summary}

Pulsar magnetospheres are natural laboratory for studying plasma physics under unusual conditionsTe.g. very strong magnetic field and highly relativistic electrons and positrons. Plasma processes in pulsar magnetospheres are not well understood $\Gamma$ in particular the processes relevant for production of pulsar radio emission. These problems continue to challenge both physicists working in astrophysics and plasma physics.

Recent discovery of eclipsing binary pulsars provides us with an opportunity to study nonlinear wave-wave interaction in electron-ion plasma in the winds (or magnetospheres) of companion stars. High brightness temperature of pulsar radio emission implies that nonlinear wave-wave interaction can be important in nonrelativistic electron-ion plasmas. Wave-wave interaction may disrupt propagation of highly beamed radio emission and result in pulsar eclipses.

\section{Acknowledgments}

The author thanks Abraham Chian for helpful discussion and FAPESP of Brazil for financial support during his visit in INPE where the work was done. Financial support of ARC through a fellowship is also acknowledged.

\section{References}

[1] R. N. Manchester and J. H. Taylor, Pulsars, (Freeman, San Francisco, 1977).

[2] F. C. Michel, Theory of Neutron Star Magnetospheres, (University Chicago Press, 1991).

[3] M. A. Ruderman and P. G. Sutherland, Astrophys. J. 196, 51 (1975).

[4] J. Arons and E. T. Scharlemann, Astrophys. J. 231, 854 (1979).

[5] J. Arons, Astrophys. J. 248, 1099 (1981).

[6] S. L. Shapiro and S. A. Teukolsky, Black Holes, White Dwarfs, and Neutron Stars, (John Wiley, New York, 1983).

[7] P. A. Sturrock, Astrophys. J. 164, 529 (1971).

[8] X. Y. Xia, G. J. Qiao, X. J. Wu and Y. Q. Hou, Astron. Astrophys. 152, 93 (1985).

[9] Q. Luo, Astrophys. J. 468, 338 (1996).

[10] P. Goldreich and W. H. Julian, Astrophys. J. 157, 869 (1969).

[11] D. B. Melrose, Aust. J. Phys. 32, 61 (1979).

[12] J. -I. Sakai and T. Kawata, J. Phys. Soc. Japan 49, 747 (1980).

[13] J. -I. Sakai and T. Kawata, J. Phys. Soc. Japan 49, $753(1980)$.

[14] M. Blaskiewicz, J. M. Cordes and I. Wasserman, Astrophys. J. 370, 643 (1991).

[15] J. Arons and J. Barnard, Astrophys. J. 302, 120 (1986).

[16] G. P. Zank and R. G. Greaves, Phys. Rev. E 51, 6079 (1995).

[17] Q. Luo and D. B. Melrose, J. Plasma Phys. 58, 345 (1997).

[18] A. S. Fruchter, D. R. Stinebring and J. H. Taylor, Nature, 333, 237 (1988).

[19] A. G. Lyne, et al., Nature 347, 650 (1990).

[20] A. G. Lyne, J. D. Biggs, P. A. Harrison and M. Bailes, Nature 361, 47 (1993).

[21] D. B. Melrose, in The Magnetospheric Structure and Emission Mechanisms of Radio Pulsars, ed. T. H. Hankins, J. M. Rankin \& J. A. Gil, (Pedagogical University Press, 1992) p. 105. 
[22] D. E. Baldwin, I. B. Bernstein and M. P. H. Weenink, Adv. Plasma Phys. 3, 1 (1969).

[23] D. B. Melrose, Plasma Astrophysics, Vol. 1, 2, (Gordon and Breach, New York, 1980).

[24] N. A. Krall and A. W. Trivelpiece, Principles of Plasma Physics, (McGraw-Hill, New York, 1973).

[25] G. Benford and R. Buschauer, Mon. Not. R. Astron. Soc. 179, 189 (1977).

[26] E. Asseo, R. Pellat and M. Rosado, Astrophys. J. 239, 661 (1983).

[27] V. V. Usov, Astrophys. J. 320, 333 (1987).

[28] S. Shibata, J. Miyazaki and F. Takahara, Mon. Not. R. Astron. Soc. 295, L53 (1998).

[29] D. B. Melrose, Astrophys. J. 225, 557 (1978).

[30] E. T. Rowe, Aust. J. Phys. 45, 1 and 45, 21 (1992).

[31] E. T. Rowe, Astron. \& Astrophys. 269, 275 (1995).

[32] E. Asseo, G. Pelletier and H. Sol, Mon. Not. R. Astron. Soc. 247, 529 (1990).

[33] A. F. Cheng and M. A. Ruderman, Astrophys. J. 212, $800(1977)$.

[34] G. Z. Machabeli and V. V. Usov, Sov. Astron. Lett. 15, 393 (1989)

[35] D. B. Melrose, Instabilities in Space and Laboratory Plasmas, (Cambridge University Press, 1986).

[36] V. V. Zheleznyakov and V. E. Shaposhnikov, Aust. J. Phys. 32, 49 (1979).

[37] Q. Luo and D. B. Melrose, Mon. Not. R. Astron. Soc. 258, 616 (1992).

[38] Q. Luo, D. B. Melrose and G. Z. Machabeli, Mon. Not. R. Astron. Soc. 268, 159 (1994).

[39] Q. Luo and D. B. Melrose, Mon. Not. R. Astron. Soc. 276, 372 (1995).

[40] C. E. Max, Phys. Fluids, 16, 1277 (1973).

[41] P. C. Clemmow, J. Plasma Phys. 12, 287 (1974).

[42] A. C. -L. Chian, Lett. Nuov. Cimento, 29, 393 (1980).

[43] X. J. Wu \& A. C. -L. Chian, Astrophys. J. 443, 261 (1995).

[44] Cordes, J. M. Space Sci. Rev. 24, 567 (1979).

[45] A. C. - L. Chian and C. F. Kennel, Astrophys. \& Space Sciences, 80, 261 (1983).

[46] A. Hasegawa, Plasma Instabilities and Nonlinear Effects (Springer-Verlage, Berlin, 1975), p. 194.

[47] R. T. Gangadhara, V. Krishan and P. K. Shukla, Mon. Not. R. Astron. Soc. 262, 151 (1993).

[48] L. Gomberoff, V. Muñoz and R. M. O. Galvão, Phys. Rev. E56, 4581 (1997).

[49] R. E. Kates and D. J. Kaup, J. Plasma Phys. 42, 521 (1989).

[50] F. T. Gratton, et al., Phys. Rev. E55, 3381 (1997).

[51] L. Gomberoff and R. M. O. Galvão, Phys. Rev. E56, 4574 (1997).
[52] V. Muñoz and L. Gomberoff, Phys. Rev. E57, 994 (1998).

[53] U. A. Mofiz, Phys. Rev. A40, 2203 (1989).

[54] U. A. Mofiz, et al. Phys. Rev. A38, 5935 (1988).

[55] L. Stenflo, P. K. Shukla and M. Y. Yu, Astrophys. Space Sci. 117, 303 (1985).

[56] F. B. Rizzato, J. Plasma Phys. 40, 289 (1988).

[57] F. B. Rizzato, R. S. Schneider and D. Dillenburg, Phys. Lett. A133, 59 (1988).

[58] A. B. Mikhailovskii, O. G. Onishchenko and E. G. Tatarinov, Plasma Phys. Contr. Fusion 27, 539 (1985).

[59] A. S. Fruchter, et al., Astrophys. J. 351, 642 (1990).

[60] A. S. Fruchter and W. M. Goss, Astrophys. J. 384, L47 (1992).

[61] M. F. Ryba and J. H. Taylor, Astrophys. J. 380, 557 (1991).

[62] Q. Luo and D. B. Melrose, Astrophys. J. 452, 346 (1995).

[63] A. S. Fruchter, J. E. Gunn, T. R. Lauer and A. Dressler, Nature 334, 686 (1988).

[64] A. S. Fruchter, J. Bookbinder and C. D. Bailyn, Astrophys. J. 443, L21 (1995).

[65] F. Arzoumanian, A. S. Fruchter and J. H. Taylor, Astrophys. J. 426, L85 (1994).

[66] D. J. Nice and S. E. Thorsett, Astrophys. J. 397, 249 (1992).

[67] D. J. Nice, S. E. Thorsett and J. H. Taylor, Astrophys. J. 361, L61 (1990).

[68] E. S. Phinney, et al. Nature 333, 823 (1988).

[69] I. Wasserman and J. M. Cordes, Astrophys. J. 333, L91 (1988).

[70] F. A. Rasio, S. L. Shapiro and S. A. Teukolsky, Astrophys. J. 342, 934 (1989).

[71] F. A. Rasio, S. L. Shapiro and S. A. Teukolsky, Astron. Astrophys. 241, L25 (1991).

[72] C. Thompson, et al. Astrophys. J. 422, 304 (1994).

[73] M. Gedalin and D. Eichler, Astrophys. J. 406, 629 (1993).

[74] D. B. Melrose, J. Plasma Phys. 51, 13 (1994).

[75] R. C. Davidson, Methods in Nonlinear Plasma Theory (Academic, New York, 1972).

[76] V. N. Tsytovich, Nonlinear Effects in Plasma (Plenum, New York, 1970).

[77] Q. Luo and D. B. Melrose, Publ. Astron. Soc. Australia 12, 71 (1995).

[78] Q. Luo and A. C.-L. Chian, Mon. Not. R. Astron. Soc. 289, 52 (1997).

[79] B. W. Stappers, et al. Astrophys. J. 465, L119 (1996).

[80] B. W. Stappers, M. S. Bessel and M. Bailes, Astrophys. J. 473, L119 (1996). 